\title{
Indicadores de desenvolvimento urbano sustentável aplicados a cidade de médio
}

\author{
porte \\ Sustainable urban development indicators applied to medium-sized city \\ Indicadores de desarrollo urbano sostenible aplicados a ciudad media
}

Recebido: 03/12/2021 | Revisado: 10/12/2021 | Aceito: 15/12/2021 | Publicado: 01/01/2022

\author{
Ivan Julio Apolonio Callejas \\ ORCID: https://orcid.org/0000-0001-7877-7029 \\ Universidade Federal do Mato Grosso, Brasil \\ E-mail: ivancallejas1973@gmail.com \\ Marcelo Martins da Cruz Neto \\ ORCID: https://orcid.org/0000-0003-2083-4021 \\ Universidade Federal do Mato Grosso, Brasil \\ E-mail: marcelo_martins1234@hotmail.com \\ Luciane Cleonice Durante \\ ORCID: https://orcid.org/0000-0002-4998-4587 \\ Universidade Federal do Mato Grosso, Brasil \\ E-mail: luciane.durante@hotmail.com \\ Emeli Lalesca Aparecida da Guarda \\ ORCID: https://orcid.org/0000-0001-7536-4448 \\ Universidade Federal de Santa Catarina, Brasil \\ E-mail: emeliguarda@gmail.com
}

\begin{abstract}
Resumo
Este trabalho tem por objetivo avaliar os indicadores de serviços urbanos e qualidade de vida para a cidade brasileira de Cuiabá-MT, por meio da aplicação da norma brasileira NBR ISO 37120, com subsequente abordagem comparativa à cidade do Rio de Janeiro-RJ, única cidade brasileira que já possui estudo semelhante. Justifica-se o estabelecimento de comparação entre comunidades para fornecer subsídios e o compartilhamento de boas práticas, contribuindo para a construção do conhecimento, ainda incipiente no âmbito brasileiro, uma vez que nenhum município brasileiro divulgou ainda oficialmente seus indicadores com base nessa normativa. A NBR ISO 37120 é baseada em 100 indicadores em 17 eixos temáticos, sendo nesta pesquisa abordados 40 indicadores em 11 eixos temáticos, a saber: Economia, Energia, Governança, Saúde, Segurança, Resíduos sólidos, Telecomunicações e Inovação, Transportes, Planejamento Urbano, Água e saneamento e Esgotos. Os resultados evidenciam que as principais diferenças entre as duas cidades são no Transporte, Planejamento Urbano, Esgotos e Água e Saneamento. Diante da importância do tema, sugere-se ao executivo municipal a utilização dos indicadores como instrumento de gestão permanente, uma vez que a quantificação auxilia na tomada de decisões municipais, permitindo avaliar as deficiências e qualidades da gestão do município.
\end{abstract}

Palavras-chave: Sustentabilidade urbana; Gestão urbana; NBR ISO 37120.

\begin{abstract}
This work aims to evaluate the indicators of urban services and quality of life for the Brazilian city of Cuiabá-MT, through the application of Brazilian code NBR ISO 37120, with a subsequent comparative approach to the city of Rio de Janeiro-RJ, the only Brazilian city that already has a similar study. The comparison establishment between communities is justified to provide subsidies and to share good practices, contributing to the construction of knowledge, which is still incipient in the Brazilian scope, since no Brazilian municipality has yet officially released its indicators based on this code. The NBR ISO 37120 is based on 100 indicators in 17 thematic axes, with 40 indicators in 11 thematic axes being addressed in this research, as followed indicated: Economy, Energy, Governance, Health, Safety, Solid Waste, Telecommunications and Innovation, Transport, Urban Planning, Water and sanitation, and Sewers. The results show that the main differences between the two cities are in Transport, Urban Planning, Sewers and Water and Sanitation. Under the importance of the theme, it is suggested to the city council the use of indicators as an instrument of permanent management, since its quantification helps in municipal decisions, allowing to assess the deficiencies and qualities of the municipality's management.
\end{abstract}

Keywords: Urban sustainability; Urban management; NBR ISO 37120.

\section{Resumen}

Este trabajo tiene como objetivo evaluar los indicadores de servicios urbanos y calidad de vida para la ciudad brasileña de Cuiabá-MT, mediante la aplicación de la norma brasileña NBR ISO 37120, con un posterior abordaje comparativo 
a la ciudad de Rio de Janeiro-RJ, el única ciudad que ya cuenta con un estudio similar. El establecimiento de comparaciones entre comunidades se justifica para otorgar subsidios y el intercambio de buenas prácticas, contribuyendo a la construcción del conocimiento, que aún es incipiente en el contexto brasileño, ya que ningún municipio brasileño ha dado a conocer oficialmente sus indicadores en base a esta normativa. La NBR ISO 37120 se basa en 100 indicadores en 17 ejes temáticos, y en esta investigación se abordan 40 indicadores en 11 ejes temáticos, a saber: Economía, Energía, Gobernanza, Salud, Seguridad, Residuos Sólidos, Telecomunicaciones e Innovación, Transporte, Urbanismo. Agua y Saneamiento y Alcantarillado. Los resultados muestran que las principales diferencias entre las dos ciudades se encuentran en Transporte, Urbanismo, Alcantarillado y Agua y Saneamiento. Dada la importancia del tema, se sugiere que el ejecutivo municipal utilice los indicadores como una herramienta de gestión permanente, ya que la cuantificación ayuda en la toma de decisiones municipales, permitiendo evaluar las deficiencias y cualidades de la gestión municipal.

Palabras clave: Sostenibilidad urbana; Gestión urbana; NBR ISO 37120.

\section{Introdução}

Atualmente, quatro bilhões de pessoas vivem em grandes cidades, e este número deve chegar a seis bilhões até 2050 (United Nations, 2019), nas quais apontam-se como os maiores problemas a crise hídrica, a ausência de saneamento e a crescente demanda por energia limpa. Apresenta-se, assim, o desafio de construir cidades mais sustentáveis, lidando com as pressões sobre os recursos básicos, como água e alimento, e utilizando processos menos bioimpactantes para prover as necessidades de mobilidade e saúde das comunidades.

Diante dessa problemática, surgem estudos propondo e utilizando-se de indicadores que expressem a realidade das comunidades, visando a mensuração de quão é sustentável seu desenvolvimento. De acordo com Loureiro et al. (2020), indicadores fornecem uma espécie de resumo ou simplificação de informações relevantes que são utilizados para fazer com que certos fenômenos que ocorrem na realidade se tornem mais aparentes. Voltando-se o olhar para essa temática, considerando-se uma janela temporal de 15 anos, é possível identificar um crescente interesse sobre o tema no Brasil: Freitas et al. (2007), Feitosa et al. (2010), Teixeira (2012), Nunes et al. (2016), Eustachio (2017), a NBR ISO 37120 (ABNT, 2017), Couto (2018) e Loureiro et al. (2020).

Freitas et al. (2007) utilizam como ferramenta para mensurar o desenvolvimento sustentável da Região do Médio ParaíbRJ, o Millenium Ecosystem Assessment (Avaliação Ecossistêmica do Milênio). Os autores estabelecem uma comparação entre os municípios objetivando identificar o andamento da sustentabilidade ambiental e o bem-estar humano na região. Para quantificação dos indicadores, foram utilizados os dados disponíveis em domínio público para sistematização de indicadores socioeconômicos e ambientais.

Feitosa et al. (2010) avaliaram o consumo de energia elétrica, combustíveis, água e a geração de resíduos sólidos na cidade de Campina Grande (PB), estabelecendo a área natural e produtiva necessária para suportar seu metabolismo. Utilizaram o Ecological Method Footprint (Método da Pegada Ecológica) que segundo Lisboa e Barros (2010) visa medir os impactos que os indivíduos de um sistema econômico provocam no sistema global, mensurando a área de ecossistema necessária para assegurar a sua sobrevivência, sendo seu cálculo importante para o estabelecimento de indicadores de desenvolvimento sustentável.

Teixeira (2012) utilizou esse mesmo método, complementando-o com o Índice de Desenvolvimento Humano (IDH) e o Índice de Gini, que abordam a esfera social e econômica, nas cidades do Rio de Janeiro, Campo Grande e Curitiba, abrangendo as temáticas de agricultura, pasto, pesca, área construída e absorção de carbono.

Nunes et al. (2016) aplicaram indicadores derivados de atributos de sustentabilidade urbana em dois bairros residenciais da cidade de Caxias do Sul - RS. Foram elaboradas matrizes de análise confrontando os conjuntos de atributos e indicadores. A partir da análise de mapas da cidade que dispunham dos atributos aplicados, foram selecionados dois bairros residenciais a serem verificados quanto à sua sustentabilidade por meio de um estudo de levantamento de dados detalhado destes locais. A metodologia aplicada aos bairros Exposição e São Pelegrino permitiu concluir que os indicadores de sustentabilidade podem ser aplicados para condições de usos diferentes, mas o caráter dos locais referenciados deve ser analisado com o cruzamento dos 
dados coletados in loco.

Eustachio (2017) utilizou como base os Objetivos do Desenvolvimento Sustentável e o método do Viable System Model, para criar o Indicador Sistêmico-Cibernético de Desenvolvimento Sustentável (ISCDS) objetivando agregar a complexidade e instabilidade que a avaliação do desenvolvimento que um município apresenta, considerando o território do estado de São Paulo. O Viable System Model é uma ferramenta organizacional que pode ser utilizado para as diversas áreas, desde gestão de empresas a municípios, com vistas a gerenciar mudanças.

Loureiro et al. (2020) indicaram a viabilidade de aplicação do método MESMIS (Marco de Evaluación de Sistemas de Manejo Incorporando Indicadores de Sustentabilidad) para medir a sustentabilidade de ambientes rurais em diferentes realidades. Os autores relatam que a metodologia tem sido aplicada nas Américas e Europa para a medição da sustentabilidade de atividades de agricultura e produção animal, com adequada comprovação do método em medir sustentabilidade em cenários distintos.

A NBR ISO 37120 (ABNT, 2017) de desenvolvimento sustentável de comunidades se pauta por uma metodologia de aplicação padronizada de indicadores para serviços urbanos e qualidade de vida, estabelecendo 100 indicadores de desenvolvimento sustentável de cidades em 17 temáticas, estruturados em três categorias. Os Essenciais e de Apoio são os requeridos e recomendados, respectivamente, para demonstrar o desempenho da prestação de serviços urbana e qualidade de vida, e os de Perfil, que tratam de estatísticas básicas que caracterizam o município, fornecendo dados relevantes para a comparação com outras cidades. Para obter a certificação normativa, a primeira categoria é obrigatória.

Essa norma não estabelece parâmetros para que se obtenha um desenvolvimento sustentável ideal, uma vez que seu objetivo é a troca de informações e boas ações entre cidades, para que a própria gestão municipal avalie o seu desempenho ao longo do tempo. Foi aplicada por Couto (2018) à cidade do Rio de Janeiro, que, em seguida, realizou a comparação com três cidades da América Latina. Justifica-se a comparação entre comunidades para fornecer subsídios à o compartilhamento de boas práticas, contribuindo para a construção do conhecimento nesse tema, incipiente no âmbito brasileiro, uma vez que nenhum município brasileiro divulgou ainda oficialmente seus indicadores com base nessa norma.

Diante do exposto, este trabalho tem por objetivo avaliar os indicadores de serviços urbanos e qualidade de vida para a cidade de Cuiabá-MT, por meio da aplicação da NBR ISO 37120 (ABNT, 2017), com subsequente abordagem comparativa à cidade do Rio de Janeiro-RJ, única cidade brasileira que já possui estudo semelhante.

\section{Metodologia}

A cidade de Cuiabá localiza-se no estado de Mato Grosso, Brasil, com área de 23.495,483km2 e densidade demográfica de 157,66hab/km2 (Tabela 1). Fundada em 1809, a cidade ficou por muito tempo isolada, passando por um processo de transformação urbana que se iniciou na década de 1930 e intensificou-se durante a década de 1960, tornando-a um dos principais centros de abastecimento para as áreas de alta produtividade agrícola da região do Cerrado e do sul da Amazônia. Em 1970, a população de Cuiabá era de 88.254 habitantes e em 2010 alcançou 551.350, um aumento de mais de 624\% (IBGE, 2017).

A pesquisa se caracteriza como quantitativa, na qual a partir de dados do município de Cuiabá-MT, aplicou-se a metodologia proposta pela NBR ISO 37120 (ABNT, 2017) de forma a estimar matematicamente os indicadores da norma (Figura 1). Os dados foram buscados nas secretarias municipais de Cuiabá, em sítios virtuais responsáveis pela divulgação dados do município e em empresas públicas e privadas que participam da gestão urbana. Na ausência de informações oficiais governamentais, utilizaram-se os disponibilizados em trabalhos científicos.

As equações dos indicadores da NBR ISO 37120 (ABNT, 2017) são de fácil aplicação e apresentam unidades de medida que facilitam a comparação, independente da cultura, economia ou forma de governo. A dificuldade da aplicação da norma está 
na disponibilidade da obtenção dos dados necessários para o cálculo dos indicadores, que não são divulgados ou monitorados usualmente pelas instituições públicas e privadas do município.

Os indicadores são divididos em de perfil, essenciais e de apoio. Os indicadores de perfil tratam de estatísticas e informações básicas que caracterizam o município, fornecendo dados relevantes para a comparação com outras cidades (Tabela 1). Já os essenciais são aqueles que são requeridos para demonstrar o desempenho da prestação de serviços urbanos e qualidade de vida, enquanto os de apoio são os recomendáveis para a demonstração do desempenho.

Tabela 1: Indicadores de Perfil para a Cidade de Cuiabá - Mato Grosso.

\begin{tabular}{|c|c|c|c|}
\hline População ${ }^{1}$ & & Habitação $^{2}$ & \\
\hline \% da população do país & $0,29 \%$ & Número total de domicílios & 188,651 \\
\hline$\%$ infantil da população (0 a 14 anos) & $22,94 \%$ & Número total de domicílios ocupados & 162,263 \\
\hline$\%$ jovem da população (15 a 24 anos) & $19 \%$ & Pessoas por domicílios & 3,32 . \\
\hline$\%$ adulta da população ( 25 a 54 anos) & $52,87 \%$ & Densidade de domicílios $\left(\mathrm{em} \mathrm{km}^{2}\right)$ & 8,029 \\
\hline$\%$ idosa da população (+65 anos) & $5,18 \%$ & & \\
\hline$\%$ da população economicamente não ativa & $52,87 \%$ & & \\
\hline Relação homem a cada 100 mulheres & $95,5 \%$ & & \\
\hline Índice de Gini ${ }^{3}$ & 0,480 & & \\
\hline Índice de Desenvolvimento Humano (IDH) ${ }^{3}$ & 0,785 & & \\
\hline
\end{tabular}

Fonte: ${ }^{1}$ (IBGE, 2017). ${ }^{2}$ (Prefeitura de Cuiabá, 2012). ${ }^{3}$ (PNUD, 2010).

Figura 1: Temáticas apresentadas na NBR ISO 37120

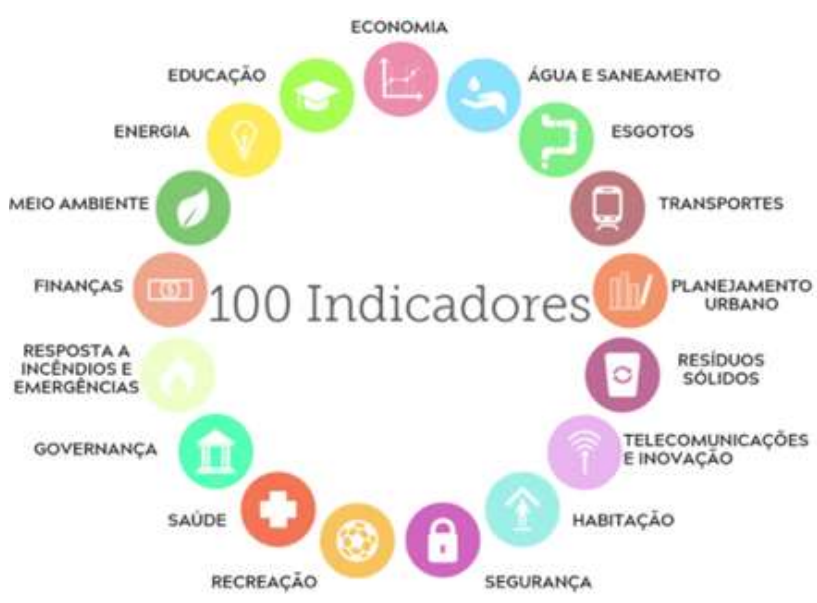

Fonte: Adaptado de WCCD (s/d).

De modo a comparar os indicadores quantificados neste trabalho, utilizou-se o levantamento conduzido por Couto (2018) no qual são apresentados os mesmos indicadores desta pesquisa para o município do Rio de Janeiro/ Estado do Rio de Janeiro. Além disso, a escolha se deu pela possibilidade de fazer a comparação do município de Cuiabá com alguma cidade brasileira, uma vez que nenhum município brasileiro divulgou ainda oficialmente seus indicadores com base na NBR ISO 37120 (ABNT, 2017).

\section{Resultados e Discussão}

Apresentam-se os resultados dos indicadores de Economia, Energia, Governança, Saúde, Segurança, Resíduos Sólidos, Telecomunicações e Inovação, Transportes, Planejamento Urbano, Esgotos e Água e Saneamento, possíveis de serem analisados a partir dos dados existentes para a cidade de Cuiabá/MT. 


\subsection{Economia}

A partir dos dados disponibilizados ou obtidos secundariamente, foi possível calcular quatro indicadores dos sete previstos nesta temática (Figura 2):

1. Taxa de desemprego na cidade (Indicador essencial);

2. Taxa de desemprego de Jovens (Indicador de Apoio);

3. Número de Empresas por 100000 habitantes (Indicador de Apoio); e,

4. Número de Novas Patentes na cidade por 100000 habitantes (Indicador de Apoio)

Figura 2: Indicadores para os Municípios de Cuiabá e Rio de Janeiro.

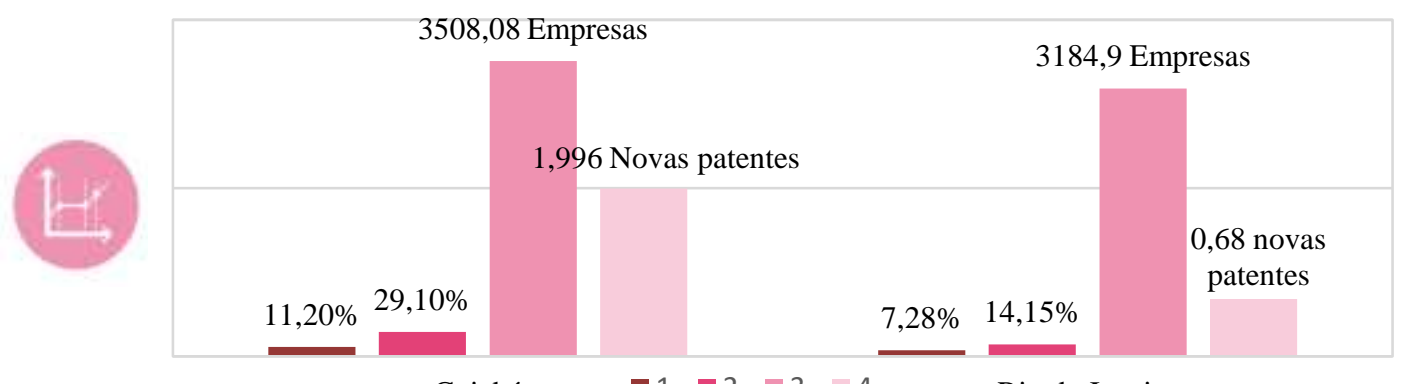

Cuiabá $\quad 1 \quad 1 \quad 2 \quad 3 \quad 4 \quad$ Rio de Janeiro

Fonte: Autores; Adaptado de Couto (2018)

Os dois primeiros indicadores do município de Cuiabá foram calculados com base no ano de 2018, o terceiro se refere a 2015 e o quarto, ao ano de 2017. Os indicadores do trabalho de Couto (2018) utilizados para comparação referem-se ao ano de 2010. A análise comparativa econômica entre Cuiabá e Rio de Janeiro se inviabiliza, pois, os momentos representam conjunturas socioeconômicas e sociais distintas.

A taxa de desemprego na cidade é considerada importante para a análise da situação do mercado de trabalho em um município. Ao comparar os dois municípios, nota-se que, em Cuiabá, a taxa é maior que no Rio de Janeiro. Segundo a NBR 37120 (ABNT, 2017), quando o crescimento econômico é forte, as taxas de desempregos tendem a ser baixas e quando a economia se encontra estagnada ou em recessão, essas taxas tendem a serem maiores. Além disso, a taxa de desemprego de Jovens é o indicador-chave para quantificar e analisar as tendências e os desafios no atual mercado de trabalho. Os dados do Número de Empresas por 100000 habitantes e do Número de Novas Patentes por 100000 habitantes foram obtidos por pesquisa em banco de dados do IBGE e do Instituto Nacional da Propriedade Industrial (INPI, 2017), respectivamente. Cuiabá possui vantagem em relação aos dois indicadores, quando comparado ao Rio de Janeiro. Isso reflete diretamente no andamento da atividade econômica no município e, também, na inovação comercial e tecnológica.

\subsection{Energia}

Dos sete indicadores de energia recomendados pela NBR ISO 37120 (ABNT, 2017), calcularam-se quatro (Figura 3), descritos a seguir, sendo os de número 1, 2 e 4 do município de Cuiabá referem-se ao ano de 2018, obtidos na concessionária de energia responsável pelo fornecimento na capital mato-grossense:

1. Uso de energia elétrica residencial per capita (kWh/ano) (Indicador essencial);

2. Porcentagem da energia total proveniente de fontes renováveis, como parte do consumo total de energia da cidade (Indicador essencial);

3. Uso total (residencial e não residencial) de energia elétrica per capita (Indicador apoio); e,

4. Duração média das interrupções de energia elétrica (em horas) (Indicador apoio). 
Figura 3: Indicadores de Energia para o município de Cuiabá e para o Rio de Janeiro.

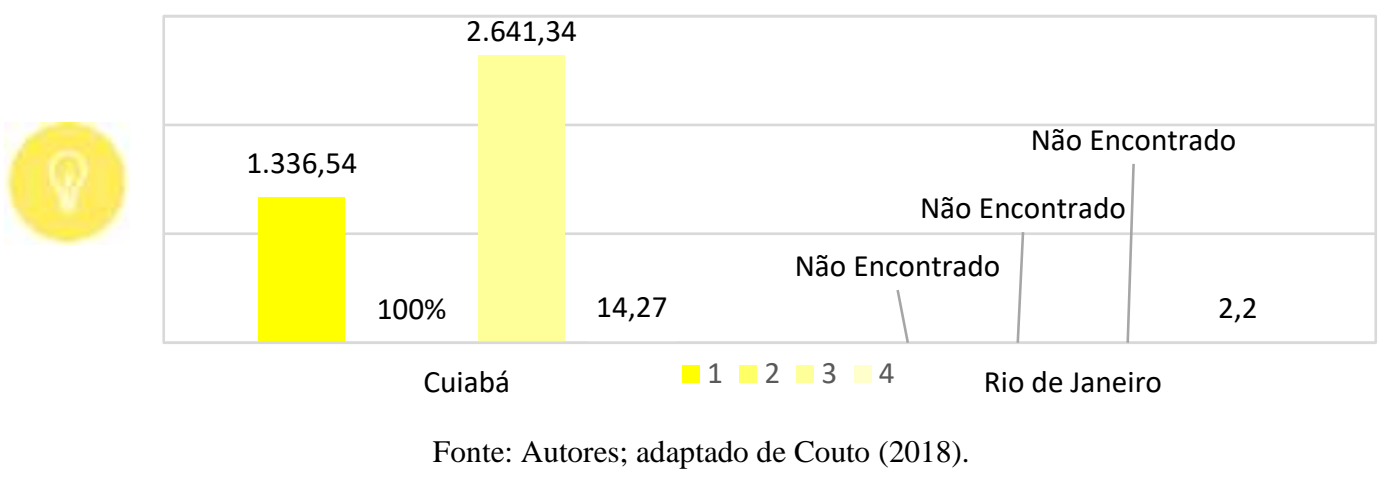

Apesar de Couto (2018) não ter divulgado os três primeiros indicadores para a cidade do Rio de Janeiro, procurou-se comparar a realidade cuiabana com a média brasileira. Ao analisar o indicador 1 - Uso total (residencial e não residencial) de energia elétrica per capita, constata-se que Cuiabá possui quantidade consumo de energia elétrica per capita de 2.6421,34 kWh por ano, bem próxima da média brasileira, que é de $2.438 \mathrm{kWh} /$ ano. Segundo The World Bank em 2014, a média brasileira de consumo de energia per capita era de 2619,961 kWh/ano. O indicador 4 - Duração média das interrupções de energia elétrica (em horas) da capital mato-grossense apresentou taxas de interrupções muito superiores a observadas no município do Rio de Janeiro, sendo $14,27 \mathrm{~h}$ no primeiro e $2,02 \mathrm{~h}$ no segundo.

O indicador 2 traz $0,22 \%$ como a porcentagem da energia total proveniente de fontes renováveis, como parte do consumo total de energia da cidade, sendo que, a realidade brasileira, $68,1 \%$ da produção de eletricidade advém de usinas hidrelétricas, consideradas fontes de energia limpa e renovável. O conceito de energia renovável está ligado às fontes que possuem a capacidade de se recuperar e nunca se esgotam, pois estão em constante renovação. As fontes renováveis são consideradas energias limpas devido à baixa agressão ao meio ambiente. Tanto a energia solar fotovoltaica, biomassa, eólica e a energia hidrelétrica são aceitas como fontes de energia renovável (EPE, 2017).

Alguns indicadores desta temática não foram encontrados tanto para o Rio de Janeiro quanto para o município de Cuiabá, em virtude da não disponibilização dos dados, uma vez que não são de mensuração usual. Como exemplo, cita-se o indicador que trata do consumo de energia por parte dos edifícios públicos $\left(\mathrm{kWh} / \mathrm{m}^{2}\right)$, que se trata de um indicador importante, uma vez que as edificações públicas são grandes consumidoras de energia nas cidades. Reduzir o consumo de energia pelos edifícios também reduz as emissões de gases de efeito estufa e sua pegada ecológica, o que pode ajudar a combater as mudanças do clima e alcançar uma economia de baixo carbono. Conforme carta resposta enviada pela concessionária de energia, em Cuiabá são consumidos $10.918 .676 \mathrm{kWh} /$ mês pelos edifícios públicos, cerca de $8,17 \%$ do consumo mensal de energia do município.

\subsection{Governança}

A temática da Governança da NBR ISO 37120 recomenda o cálculo de seis indicadores, dentre os quais foram calculados dois (Figura 4):

1. Porcentagem de participação dos eleitores nas últimas eleições municipais em função do total de eleitores aptos a votar (Indicador Essencial) e;

2. Porcentagem de mulheres eleitas em função do número total de eleitos na gestão da cidade (Indicador essencial). 
Figura 4: Indicadores de Governança para Cuiabá e para o Rio de Janeiro.

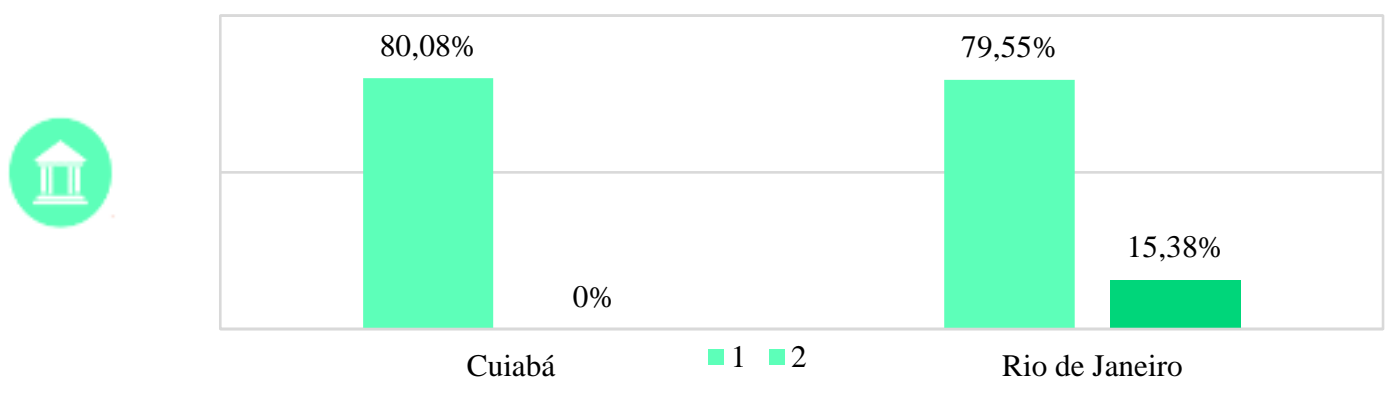

Fonte: Autores; adaptado de Couto (2018).

A última eleição municipal em Cuiabá foi realizada no ano de 2016 e contou com participação de $80,08 \%$ dos aptos a votar. Nota-se que os dois municípios possuem valores próximos, no entanto, o indicador 2 revela divergência entre a governança dos municípios. Na eleição de 2016 foram eleitos um prefeito e um vice-prefeito para o poder executivo e 25 vereadores, sendo que entre os eleitos, não foi eleita nenhuma mulher para a gestão do município. A divergência é preocupante, pois a porcentagem de mulheres eleitas para cargos da gestão da cidade é um reflexo direto da inclusão na governança.

\subsection{Saúde}

Dos sete indicadores de energia recomendados pela NBR ISO 37120, calcularam-se cinco (Figura 5), descritos a seguir:

1. Expectativa Média de Vida (Indicador Essencial);

2. Número de Leitos hospitalares por 100.000 habitantes (Indicador Essencial);

3. Número de Médicos por 100.000 habitantes (Indicador Essencial);

4. Taxa de Mortalidade de crianças menores de cinco anos a cada 1000 nascidos vivos (Indicador essencial); e,

5. Taxa de Suicídio por 100.000 habitantes.

Figura 5: Indicadores de Saúde para Cuiabá e para o Rio de Janeiro.

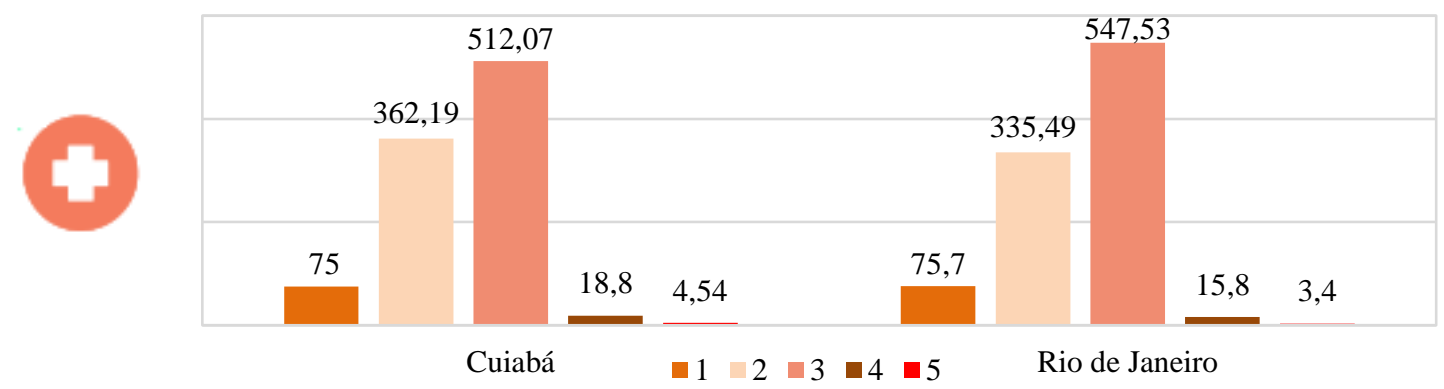

Fonte: Autores; adaptado de Couto (2018).

A expectativa de vida e consequentemente o nível de mortalidade de uma população está intimamente ligada às condições de saúde, que são uma parte integrante do desenvolvimento (ABNT, 2017). Sendo assim, ao analisar o indicador 1 para as duas cidades, nota-se que as expectativas de vida possuem valores bem próximos. O mesmo ocorre para os indicadores 2 e 3, e apesar de Cuiabá possuir maior quantidade de leitos por 100.000 habitantes, a cidade possui número inferior de médicos por 100.000 habitantes, se comparado ao Rio de Janeiro. O indicador 5 é de grande importância pois aponta o nível de saúde infantil e desenvolvimento global nas cidades. Além disso, demostra o quanto a cidade é um lugar saudável ou não para se viver, sendo um dos Objetivos de Desenvolvimento (ONU, 2015). 
A taxa de suicídio em Cuiabá é de 4,54 suicídios a cada 100.000 habitantes (indicador 4), superando a taxa da cidade do Rio de Janeiro. Trata-se de um indicador importante, pois reflete na saúde mental da cidade, fator esse determinante para o desenvolvimento humano.

\subsection{Segurança}

A temática da Segurança recomenda o cálculo de cinco indicadores, porém, com os dados disponibilizados, foi possível calcular somente o Número de Homicídios por 100.000 habitantes (Indicador Essencial), indicado pela legenda 1 na Figura 6.

Figura 6: Indicadores de Segurança para Cuiabá e para o Rio de Janeiro.

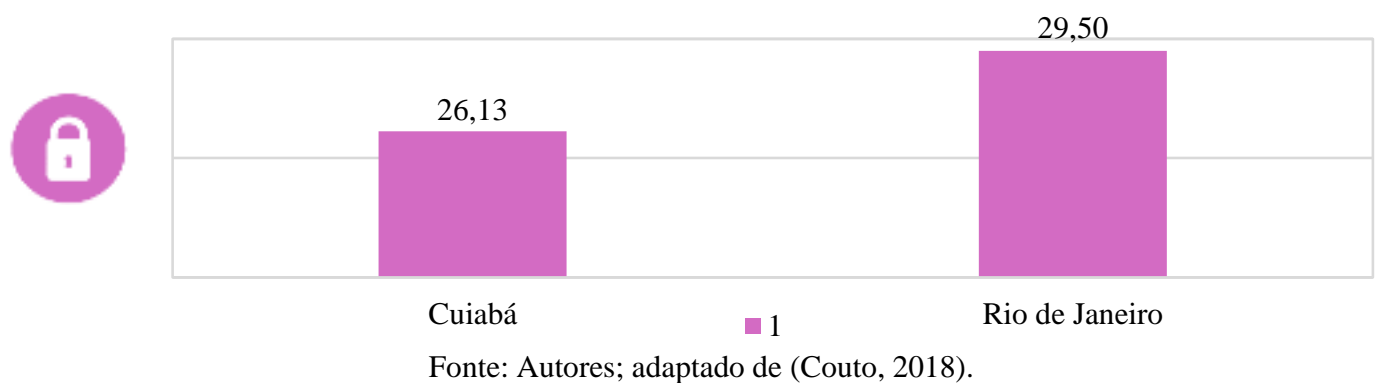

O número de homicídios é um indicador de quantidade de crimes e reflete a percepção de segurança pessoal, podendo afetar os incentivos aos investimentos (ABNT, 2017). Constata-se que o município de Cuiabá possui menor taxa de homicídio em relação ao Rio de Janeiro, sendo as taxas consideradas elevadas se comparadas a aquelas de países desenvolvidos.

\subsection{Resíduos Sólidos}

A temática dos Resíduos Sólidos da NBR ISO 37120 recomenda o cálculo de dez indicadores, porém, com os dados disponibilizados, foram avaliados oito (Figura 7), a saber:

1. Porcentagem da população urbana com coleta regular de resíduos sólidos (Essencial);

2. Total de coleta de resíduos sólidos municipais per capita (Indicador Essencial);

3. Porcentagem de resíduos sólidos urbanos que são reciclados (Indicador Essencial);

4. Porcentagem de resíduos sólidos urbanos dispostos em aterros sanitários (Indicador apoio);

5. Porcentagem de resíduos sólidos urbanos descartados para incineração (Indicador Apoio);

6. Porcentagem de resíduos sólidos urbanos queimados a céu aberto (Indicador Apoio);

7. Porcentagem de resíduos sólidos urbanos em lixões a céu aberto (Indicador Apoio); e,

8. Porcentagem de resíduos sólidos urbanos dispostos em outros meios (Indicador Apoio).

Figura 7: Indicadores de Resíduos Sólidos para Cuiabá e para o Rio de Janeiro.

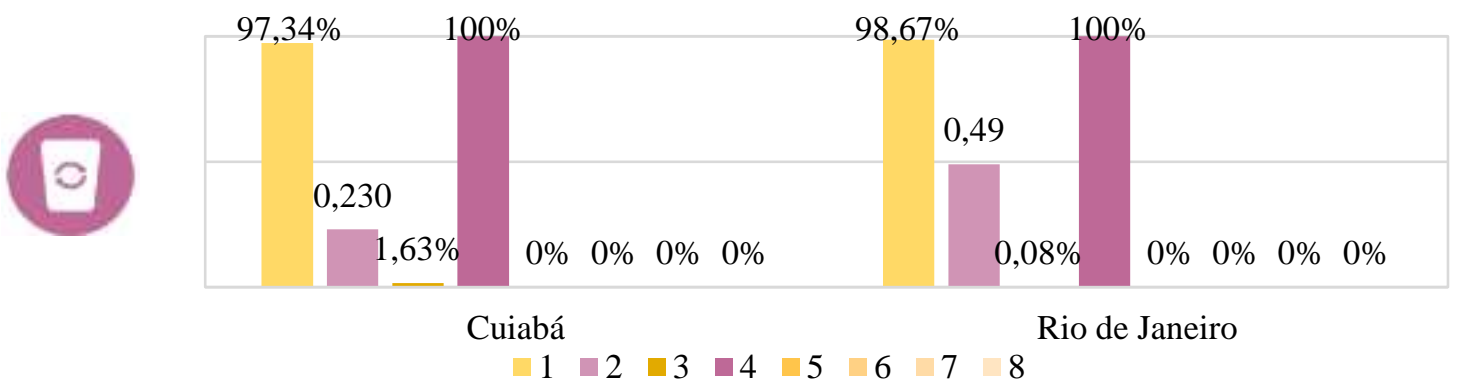

Fonte: Autores; adaptado de Couto (2018). 
A porcentagem da população urbana com coleta regular de resíduos quantificada por meio do indicador 1 aponta um elevado alcance da administração pública em relação ao atendimento. Ambas as cidades se assemelham nesse quesito e atingem quase a totalidade da população com a coleta domiciliar, o que contribui para o meio ambiente e para a saúde pública urbana. $\mathrm{O}$ lixo acumulado em bairros pode gerar alagamentos e facilitar o acúmulo de água e, com proliferação de mosquitos transmissores de doenças, problemática comum as duas cidades. Nesse sentido, a gestão dos resíduos sólidos é uma importante estratégia para evitar que tais problemas ambientais não ocorram. Santos et al. (2021) adotaram como medida mitigadora no município de São José do Seridó/RN a implantação do aterro controlado, coleta seletiva, ações educativas nas escolas sobre o descarte do lixo, entre outros com vista e evitar o descarte em locais inapropriados.

$\mathrm{O}$ indicador 2 retrata da quantidade total de resíduos per capita nos municípios e revela que o Rio de Janeiro apresenta mais que o dobro da quantidade de lixo per capita do que o município de Cuiabá, indicando dois possíveis aspectos: que a coleta de lixo na cidade é mais efetiva ou ainda possível maior renda da população, refletindo em maior consumo de bens.

Ao analisar o Indicador 3, que se refere a porcentagem de resíduos reciclados, constata-se que ambas as cidades pesquisadas apresentam baixa taxa de reciclagem, com maior percentagem de resíduos destinados para reaproveitamento na cidade Cuiabá. Isso indica que a Lei Federal 12.305 (Brasil, 2010) que institui a Política Nacional dos Resíduos Sólidos não está cumprindo sua função. Aponta-se para a necessidade de ambos os municípios em incentivar a reciclagem de resíduos.

O indicador 4 indica que 100\% dos resíduos sólidos estão sendo encaminhados para aterros sanitários, não retratando a realidade observada nas cidades brasileiras, uma vez que, em ambas as cidades, é possível encontrar resíduos dispostos de forma irregular. Nos municípios não há a queima de resíduos por incineração, necessária em cidades que geram mais resíduos sólidos do que podem dispor. Além disso, ambos não dispõem resíduos a céu aberto e/ou lixões. As respostas às solicitações informaram que disposição em tais locais é proibida pela Lei Federal 12.305 (Brasil, 2010). A coleta de resíduos perigosos em ambas as cidades é feita por empresas privadas, com dados não disponibilizados, sob alegação de confidencialidade.

\subsection{Telecomunicações e Inovação}

A temática de Telecomunicações e Inovação da NBR ISO 37120 recomenda o cálculo de três indicadores (Figura 8):

1. Número de Conexões de Internet por 100.000 habitantes (Indicador Essencial);

2. Número de conexões de telefone celular por 100.000 habitantes (Indicador Essencial); e,

3. Número de conexões de telefone fixo por 100.000 habitantes (Indicador Essencial).

Figura 8: Indicadores de Telecomunicações para Cuiabá e para o Rio de Janeiro.

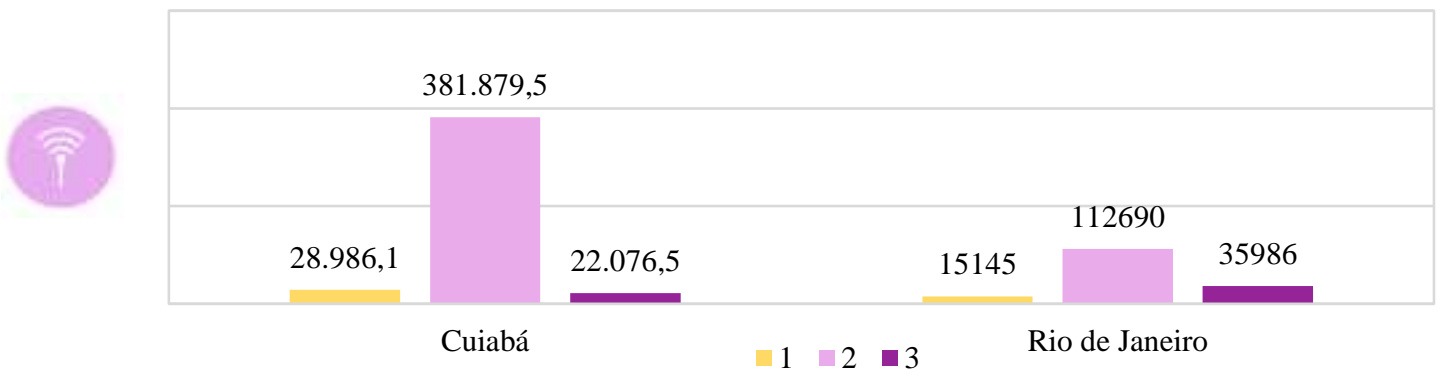

Fonte: Autores; adaptado de Couto (2018).

O número de conexões de internet é um indicador de acesso à informação e conectividade à tecnologia de comunicação. Como os dados para Cuiabá são do ano de 2018 e os dados para o Rio de Janeiro são para o ano de 2010, é razoável que o Rio de Janeiro apresente taxa inferior. É notável que o indicador 2 ultrapassa 1,0, isso porque ele foi calculado utilizando o total de 
telefones com DDD 65, ao invés de se utilizar o número de telefones pertencentes apenas ao município de Cuiabá. Ao analisar o indicador 3, constata-se que mesmo se tratando do ano de 2010, o Rio de Janeiro possui número elevado de telefones fixos por 100.000 habitantes.

\subsection{Transportes}

A temática dos Transportes da NBR ISO 37120 recomenda o cálculo de nove indicadores, porém, com os dados disponíveis, foram calculados seis (Figura 9):

1. Quilômetros de Transporte Público de alta capacidade (Indicador Essencial);

2. Número anual de viagens em transporte público per capita (Indicador Essencial);

3. Número de automóveis privados per capita (Indicador Essencial);

4. Número de veículos motorizados de duas rodas per capita (Indicador de Apoio);

5. Quilômetros de Ciclovia e ciclo faixas por 100.000 habitantes; e,

6. Mortalidade de Trânsito por 100.000 habitantes.

Figura 9: Indicadores de Transporte para Cuiabá e para o Rio de Janeiro.

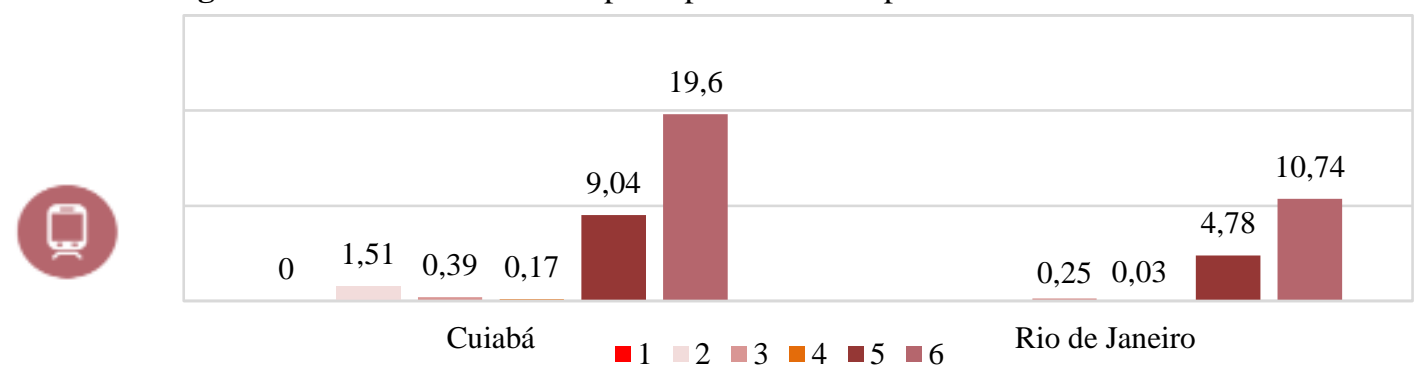

Fonte: Autores; adaptado de Couto (2018).

Os indicadores de transportes representam como se desenvolve a logística na cidade, sendo de suma importância, pois o transporte influencia na economia e no desenvolvimento humano. Ainda, a ineficiência do sistema de transporte público é um os principais fenômenos da mobilidade urbana enfrentados diariamente pelas populações das cidades tradicionais em todo o mundo, devendo os setores público e privado encontrar soluções urgentes e viáveis com vistas a melhorar a qualidade de vida da população (Queiroz et al. 2021). Ao analisar o indicador 1 e 2, nota-se que Cuiabá não possui transporte público de alta capacidade. Só há funcionamento de sistema de ônibus, com 1,51 viagens per capita no ano. O Rio de janeiro não possui dados divulgados para esses indicadores. Em relação ao indicador 3, nota-se que em Cuiabá a taxa automóvel per capita é superior à do Rio de Janeiro, não só pelo fato da população do Rio de Janeiro ser superior ao número de automóveis, mas também porque parte da população vive em favelas, onde a mobilidade é reduzida. O indicador 4 trata dos veículos de duas rodas, com utilização desse tipo de veículo per capita em Cuiabá muito superior ao Rio de Janeiro. Apesar do Rio de Janeiro possuir maior extensão de ciclovias e ciclo faixas ainda possui uma quantidade inferior por 100.000 habitantes quando comparado com Cuiabá. Quanto à mortalidade no trânsito, as taxas cuiabanas são superiores as fluminenses.

\subsection{Planejamento Urbano}

A temática de Planejamento Urbano da NBR ISO 37120 recomenda o cálculo de quatro indicadores, porém, com os dados disponibilizados, foram calculados dois (Figura 10):

1. Áreas verdes (hectares) por 100.000 habitantes (Indicador Essencial); e,

2. Relação emprego/habitação (Indicador Essencial). 
Figura 10: Indicadores de Planejamento para Cuiabá e para o Rio de Janeiro.

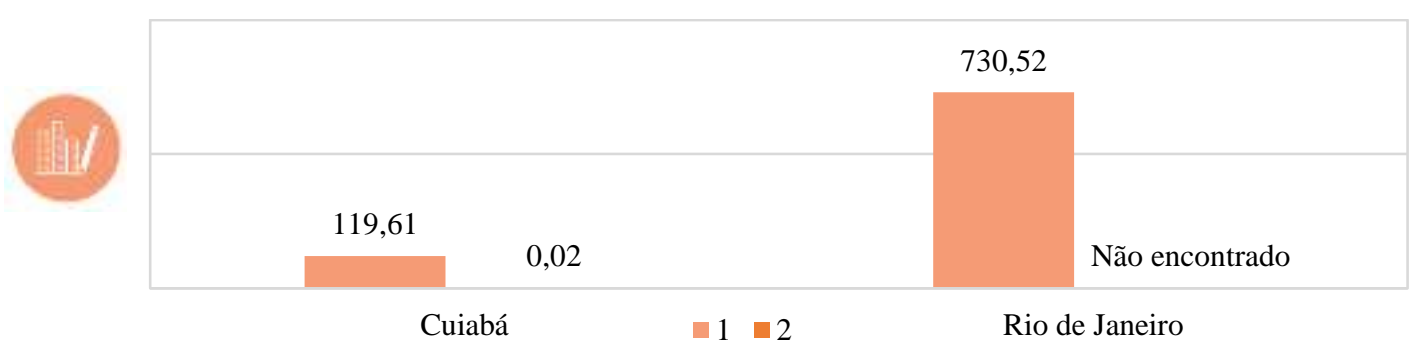

Fonte: Autores; adaptado de Couto (2018).

É importante salientar que o município de Cuiabá se encontra no Cerrado brasileiro, bioma este característico por porte arbóreo inferior ao de grandes florestas como a Amazônia ou a Mata Atlântica. Já o Rio de Janeiro se encontra inserido na Mata Atlântica, possuindo florestas mais densas e úmidas. Além disso, é necessário observar que o Rio de Janeiro possui regiões de difícil exploração por possuir um relevo comporto por cadeia montanhosa, enquanto em Cuiabá, este é caracterizado por região de depressão. Assim, devido ao acesso, o Rio de Janeiro acaba possuindo uma maior quantidade de hectares de área verde por 100.000 habitantes, devido as grandes áreas preservadas junto a cadeia montanhosa. Segundo ABNT (2017), a quantidade de áreas verdes, parques naturais e seminaturais e demais espaços abertos é um indicador importante, pois estas realizam funções ambientais em um ambiente urbano, melhorando o clima, capturando poluentes e melhorando a qualidade de vida. Sob este aspecto, várias propostas podem ser trazidas do estudo de Silva et al. (2020) para a qual na cidade de Salvador foram propostas ações para melhoria desse indicador como Recuperação Paisagística, desenvolvimento de Plano Diretor de Arborização Urbana, Manual Técnico de Arborização de Salvador, Programa de Arborização Participativa (PAP), entre outros.

O indicador 2 que trata a relação emprego/habitação e reflete a quantidade de postos de trabalho disponíveis no município em relação aos domicílios existentes.

\subsection{Esgotos}

A temática de Esgotos da NBR ISO 37120 recomenda o cálculo de cinco indicadores, porém, com os dados disponibilizados, calculou-se dois (Figura 11), sendo esses apresentados abaixo:

1. Porcentagem da população urbana atendida por sistemas de coleta e afastamento de esgoto (Indicador Essencial); e,

2. Porcentagem de coleta do esgoto da cidade que não recebeu qualquer tratamento (Indicador Essencial).

Os dados foram coletados do Sistema Nacional de Informação de Saneamento (SNIS, 2018).

Figura 11: Indicadores de Esgotos para Cuiabá e para o Rio de Janeiro.

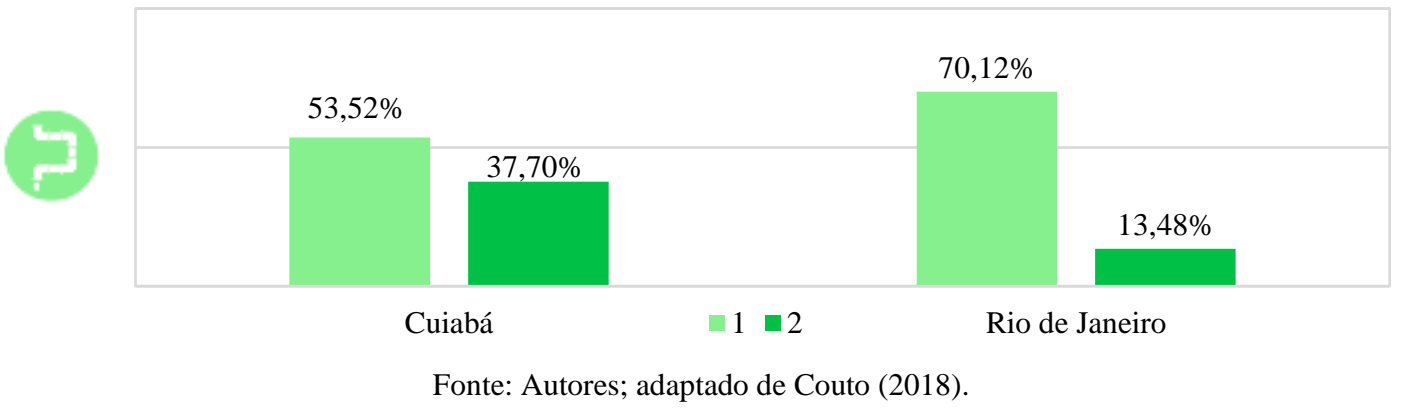

É possível analisar que quase $50 \%$ da população cuiabana não é atendida por sistemas de coleta e afastamento de esgoto. Este indicador revela a deficiência da gestão municipal em atender a população. Esta deficiência influencia diretamente nas 
outras questões inerentes ao desenvolvimento sustentável, como saúde e qualidade de vida. Além disso, é possível perceber que a quantidade de esgoto tratado ainda é muito baixa $(37,70 \%)$, indicando que grande parcela do esgoto não é coletada e nem tratada, sendo descartado em rios e córregos da cidade. Esse indicador indica a necessidade de políticas públicas voltadas para a questão do saneamento básico. Um sistema de tratamento de esgoto confiável é o principal indicador do nível de desenvolvimento local e de saúde da comunidade (ABNT, 2017). A cidade do Rio de Janeiro apresenta-se mais evoluída na coleta e tratamento de resíduos.

\subsection{1 Água e Saneamento}

A temática de Água e Saneamento da NBR ISO 37120 recomenda o cálculo de sete indicadores, porém, com os dados disponíveis, foram calculados apenas quatro (Figura 12):

1. Porcentagem da população com serviço de abastecimento de água potável (Essencial);

2. Consumo total de água per capita (litros por dia) (Indicador Essencial); e,

3. Porcentagem de perdas de água (água não faturada) (Indicador Apoio).

Os dados foram coletados do Sistema Nacional de Informação de Saneamento (SNIS, 2018).

Figura 12: Indicadores de Esgotos para Cuiabá e para o Rio de Janeiro.

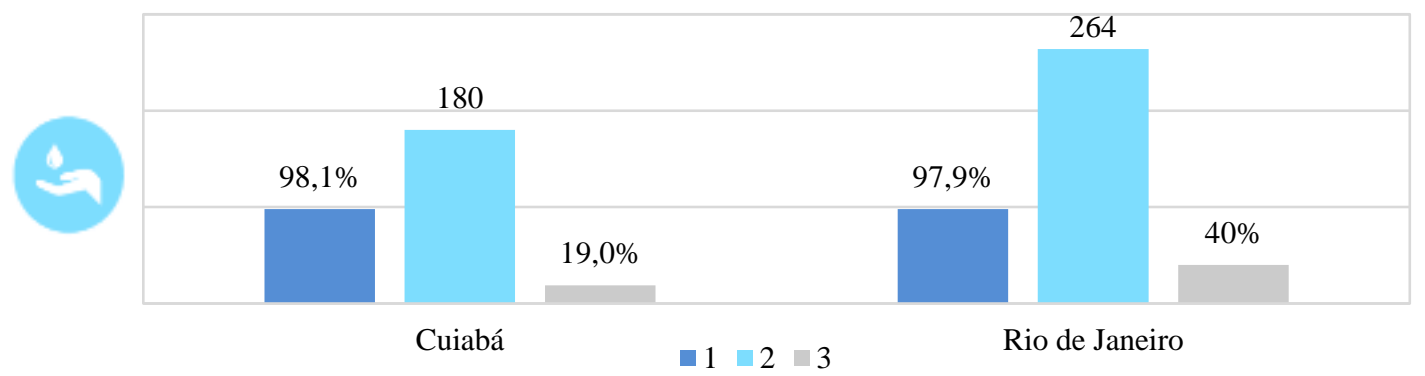

Fonte: Autores; adaptado de Couto (2018).

É possível constatar que ambos os municípios possuem valores próximos de atendimento à população. A porcentagem alta demonstra que a gestão municipal está sendo eficiente no abastecimento de água potável. Importante ressaltar que esse é um indicador que permite aferir a saúde e a qualidade de vida na cidade. O indicador 2 refere-se ao consumo total de água per capita por dia e ao se comparar a cidade do Rio de Janeiro com Cuiabá, constata-se que, que apesar de condições climáticas semelhantes, e questões de preço e de destinação e uso diferentes, o consumo de água em Cuiabá é inferior, mostrando que o padrão de consumo de água do município é, de certa forma, mais sustentável. O consumo de água deve estar em harmonia com os recursos hídricos para ser sustentável (ABNT, 2017).

O indicador 3 refere-se porcentagem de água não faturada no município. As perdas nas redes de distribuição de água são fatores relevantes que devem ser observados pela gestão municipal. Constata-se que em Cuiabá a perda de água é inferior, demonstrando assim maior precaução por parte da concessionária de água local no controle das perdas.

\section{Conclusão}

Pesquisaram-se 40 indicadores, consolidados em 11 eixos temáticos, o que revela a deficiência da gestão pública municipal em produzir informações aos seus munícipes. Em parte, pode-se atribuir a escassez de informações ao fato de a norma ser recente, publicada no ano de 2017. Destaca-se que os indicadores são de extrema relevância para análise da sustentabilidade do município e seu cálculo é necessário para que Cuiabá seja registrada em redes de cidades que divulgam seus indicadores, a 
exemplo da plataforma WORLD CONCIL ON CITY DATA (WCCD, s/d). Entre as temáticas não abordadas nessa pesquisa, cita-se a dos eixos de Educação, Meio Ambiente, Finanças, Resposta a Incêndios e Emergências, Recreação e Habitação.

Em relação à temática Economia, pode-se inferir que, apesar da taxa de desemprego estar alta, resultado da atual crise econômica por que passa o país, nota-se que os indicados se apresentam satisfatórios em relação ao número de empresas e novas patentes, ou seja, a inovação tecnológica e movimentação do mercado estão presentes no município.

Quanto aos indicadores de Energia, constata-se que o consumo per capita por ano na cidade de Cuiabá está próximo a média nacional brasileira. Além disso, a totalidade de energia é provida por meio de hidrelétricas, considerada fonte renovável.

Sob o aspecto da Governança, conclui-se que o os cidadãos cuiabanos têm grande participação na escolha de seus líderes municipais. Porém, não há participação feminina na gestão municipal, sendo um fator preocupante em relação a inclusão feminina na participação das decisões, uma vez que, 51,2\% da população cuiabana é composta por mulheres (PNUD, 2010).

Em relação à temática da Saúde, Cuiabá apresentou valores de indicadores bem próximos a aqueles quantificados na cidade do Rio de Janeiro. Porém, apresenta resultados insatisfatórios quanto à taxa de suicídio e mortalidade infantil, o que orienta a gestão municipal em prover maior atenção e enfoque na saúde mental da população e no cuidado com a saúde infantil.

Os indicadores de Segurança são alarmantes nas duas cidades, demonstrando a necessidade de políticas públicas mais efetivas visando reduzir as taxas de homicídios, muito embora a taxa em Cuiabá seja menor que a do Rio de Janeiro.

Em relação aos indicadores de Resíduos sólidos, verifica-se que a cidade de Cuiabá possui uma porcentagem satisfatória em relação ao atendimento da população na coleta de resíduos sólidos. A quantidade total de resíduos per capita foi quase o dobro inferior ao do Rio de Janeiro, indicando dois possíveis aspectos: que a coleta de lixo na cidade é mais efetiva, não havendo disposição em lixões ou locais clandestinos ou ainda uma possível maior renda da população, o que se reflete em maior consumo de bens. Também se constatou que o município obedece às legislações vigentes e destina seu lixo apenas a aterros sanitários, conforme informado repassada pelo órgão municipal competente. Além disso, possui regularidade em relação à Política Nacional dos Resíduos Sólidos dentro dos indicadores de incineração, descarte por queimada e disposição em lixões.

Quanto à temática de Telecomunicações e Inovação, pode-se concluir que o município possui grande quantidade de conexões de internet, telefone celular e fixo, o que demonstra que há conectividade local e global, contribuindo significativamente para o crescimento econômico e o desenvolvimento tanto regional quanto nacional da capital mato-grossense.

Em relação ao eixo de Transporte, registra-se que Cuiabá possui transporte público com baixa quantidade de viagens per capita ao ano. Outro aspecto relevante é que a taxa de mortalidade no trânsito se apresenta elevada, sendo este um fator que pode ser revertido com maior fiscalização e rigor dos agentes públicos além de investimento em educação no trânsito.

Quanto ao Planejamento urbano, Cuiabá se mostrou deficiente na quantidade de áreas verdes, o que influencia no microclima urbano e na qualidade de vida da população, sendo assim, a gestão municipal precisa investir em plantio de árvores e construção de parques.

As duas últimas duas temáticas tratam de Água e Saneamento e Esgotos. No que tange a esse eixo, o município se mostrou eficiente na distribuição de água e no controle de suas perdas. Entretanto, apresentou deficiência em gerir os resíduos líquidos provenientes dos esgotos, uma vez que a maioria deste não é tratada, sendo lançado diretamente em rios e córregos do município, prejudicando os ecossistemas aquáticos e também a saúde da população.

Dessa forma, a aplicação da NBR ISO 37120 (ABNT, 2017) à cidade de Cuiabá possibilitou a quantificação de informações relevantes aos gestores da capital mato-grossense. O trabalho limitou-se aos indicadores possíveis de serem analisados com só dados existentes, tendo, assim seu escopo limitado à disponibilidade dos dados: dos 100 indicadores em 17 eixos temáticos da norma, foram abordados 40 indicadores em 11 eixos temáticos. Assim, trabalhos futuros que possam ampliar a análise a partir da busca de novos dados são recomendados, bem como o aprofundamento das análises de cada eixo temático, à luz das políticas existente no município de Cuiabá, visando a proposição de ações de desenvolvimento sustentável. Diante da 
importância do tema tratado nesta pesquisa, sugere-se ao executivo municipal a utilização dos indicadores como instrumento de gestão permanente, uma vez que a quantificação auxilia na tomada de decisões municipais, permitindo avaliar as deficiências e qualidades da gestão do município.

\section{Referências}

Associação Brasileira de Normas Técnicas (2017). ABNT NBR ISO 37120. Desenvolvimento sustentável de comunidades - Indicadores para serviços urbanos e qualidade de vida. https://www.abntcatalogo.com.br/norma.aspx?ID=366389

Brasil (2010). Lei Federal $n^{\circ}$ 12.305, de 2 de agosto de 2010. Institui a Política Nacional de Resíduos Sólidos; altera a Lei no 9.605 , de 12 de fevereiro de 1998; e dá outras providências [online]. Brasília: Diário Oficial da União. http://www.planalto.gov.br/ccivil_03/_ato2007-2010/2010/lei/112305.htm

Couto, E. A (2018). Aplicação dos indicadores de desenvolvimento sustentável da norma NBR $37120: 2017$ para a cidade do Rio de Janeiro e análise comparativa com cidades da América Latina [online]. Monografia. UFRJ. http://www.monografias.poli.ufrj.br/monografias/monopoli10024924.pdf

Empresa de Pesquisa Energética (2017). Estudos de Planejamento da Expansão da Geração: Avaliação da Geração [online]. Brasília: Editora do Governo Federal. https://www.epe.gov.br/sites-pt/publicacoes-dados-abertos/publicacoes/PublicacoesArquivos/publicacao-232/topico-214/Metodologia\%20para\% 20avalia\%C3\%A7\%C3\%A3o\%20de\%20usinas\%20h\%C3\%ADbridas\%20e\%C3\%B3lico-fotovoltaicas.pdf

Eustachio, J. H. P. P. (2017). A estrutura do sistema viável de desenvolvimento sustentável a partir dos objetivos de desenvolvimento sustentável da ONU para os municípios do estado de São Paulo: uma aplicação do Viable System Model para prefeituras municipais. Mestrado. USP, 2017. https://www.teses.usp.br/teses/disponiveis/96/96132/tde-06102017-160957/pt-br.php

Feitosa, M. J. S., Cândido, G. A., Firmo, L. A. (2010). Sistemas de Indicadores de Sustentabilidade: uma aplicação do Ecological Footprint Method no município de Campina Grande (PB). Ambiência 6(3), 393 - 414. https://revistas.unicentro.br/index.php/ambiencia/article/view/960

Freitas, C. M., Schütz, G. E., Oliveira, S. G. (2007). Environmental sustainability and human well-being indicators from the ecosystem perspective in the Middle Paraíba Region, Rio de Janeiro State, Brazil. Cad. Saúde Pública 23(4), S513-S528. http://dx.doi.org/10.1590/S0102-311X2007001600012.

Instituto Brasileiro de Geografia e Estatística - IBGE (2017). Estimativas Populacionais 2017 www.ibge.gov.br.

Instituto Brasileiro de Geografia e Estatística - IBGE (s/d). PNAD-CONTÍNUA. IBGE. [www.ibge.gov.br.

Instituto Nacional de Propriedade Industrial - INPI (2017). Anuário Estatístico de Propriedade Industrial: 2000-2012 http://www.inpi.gov.br/sobre/estatisticas/anuario-estatistico-de-propriedadeindustrial-2000-2012-patente2\#patente.

Lisboa, C., K., Barros, M., V., F. (2010). A pegada ecológica como instrumento de avaliação ambiental para a cidade de Londrina. Confins, 8, https://doi.org/10.4000/confins.6395

Loureiro, J. P. B. de; Santos, M. A. S. dos; Rodrigues, H. E.; Souza, C. C. F. de; Rebello, F. K (2020). Evaluation of natural resource management systems based on sustainability indicators: A systematic literature review of MESMIS method use. Research, Society and Development, 9(8), e538986067. 10.33448/rsdv9i8.6067. https://rsdjournal.org/index.php/rsd/article/view/6067.

Nunes, M., F., O., Mayorga, C., T., Rosa Gullo, M., C., Mesquita Pedone, C., E. (2016). Indicadores de sustentabilidade urbana: Aplicação em bairros de Caxias do Sul. Arquiteturarevista 12(1), 87-100. https://doi.org/10.4013/arq.2016.121.08

Organização das nações unidas - ONU (2015). Transformando Nosso Mundo: A Agenda 2030 para o Desenvolvimento Sustentável https://nacoesunidas.org/pos2015/agenda2030/.

Prefeitura de Cuiabá (2012). Perfil Socioeconômico de Cuiabá. Vol. V. Instituto de Planejamento e Desenvolvimento Urbano (IPDU), Diretoria de Pesquisa e Informação (DPI). Cuiabá: Central de Texto.: https://www.cuiaba.mt.gov.br/upload/arquivo/20130117130637.zip

Programa das Nações Unidas para o Desenvolvimento - PNUD (2010). Instituto De Pesquisa Econômica Aplicada - IPEA, Fundação João Pinheiro; Instituto Brasileiro De Geografia E Estatística - IBGE. 2010. Atlas do Desenvolvimento Humano no Brasil https://1drv.ms/u/s!AuwEBHxVU0YSgbJeae6tWqJzHlKf4w?e=92fqJK

Queiroz, A. P. F. de; Guimarães Júnior, D. S.; Nascimento, A. M. .; Melo, F. J. C. (2021). Overview of Urban Mobility in Smart Cities. Research, Society and Development, 10(9), e18210917830. 10.33448/rsd-v10i9.17830. https://rsdjournal.org/index.php/rsd/article/view/17830.

Santos, K. L. de A.; Medeiros, J. A. de .; Queiroz, L. M. N. de .; Araujo, P. P. D. G. de .; Fontes Júnior, D. F. (2021). Urban solid waste and the 2030 Agenda: An analysis of actions taken by the municipality of São José do Seridó/RN. Research, Society and Development, 10(7), e3410716205. 10.33448/rsd-v10i7.16205. Disponível em: https://rsdjournal.org/index.php/rsd/article/view/16205.

Silva, P. P.; Araújo, P. S. R. (2020). Sustainable urban actions in Salvador City, Bahia State, Brazil (2013-2016). Research, Society and Development, 9(8), e319984976. 10.33448/rsd-v9i8.4976. Disponível em: https://rsdjournal.org/index.php/rsd/article/view/4976.

Sistema Nacional de Informações sobre Saneamento - SNIS (2018). Série Histórica. Brasília: Ministério das Cidades http://app4.cidades.gov.br/serieHistorica Teixeira, M., F., B. (2012). Pegada Ecológica e Políticas Públicas: estudos de caso de três cidades brasileiras. Revibec: revista iberoamericana de economía ecológica 19, 15-28, https://www.raco.cat/index.php/Revibec/article/view/261788

The World Bank. Electric Power Consumption https://data.worldbank.org/indicator/EG.USE.ELEC.KH.PC?locations=BR 
Research, Society and Development, v. 11, n. 1, e1111124333, 2022

(CC BY 4.0) | ISSN 2525-3409 | DOI: http://dx.doi.org/10.33448/rsd-v11i1.24333

United Nations (2019). Department of Economic and Social Affairs, Population Division (2019). World Urbanization Prospects: The 2018 Revision (ST/ESA/SER.A/420 [online]). United Nations. https://population.un.org/wup/Publications/Files/WUP2018-Report.pdf.

World Concil on City Data - WCCD (s/d). Compare Cities Worldwide. https://www.dataforcities.org/ 